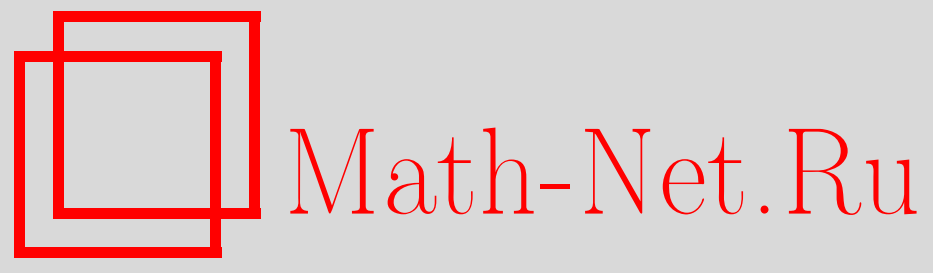

А. В. Нехожин, Двухслойная математическая модель шейки бедра человека для исследования напряжённого состояния при армировании имплантатами различной конструкции, Вестн. Сам. гос. техн. ун-та. Сер. Физ.-мат. науки, 2013, выпуск 3(), 129-135

DOI: https://doi.org/10.14498/vsgtu1254

Использование Общероссийского математического портала MathNet.Ru подразумевает, что вы прочитали и согласны с пользовательским соглашением

http://www . mathnet.ru/rus/agreement

Параметры загрузки:

IP : 52.87 .193 .239

26 апреля 2023 г., 12:01:45

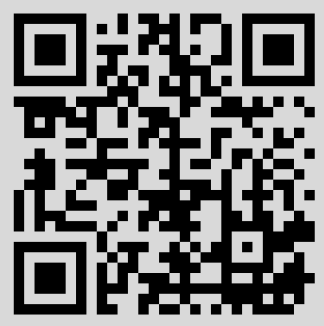


УДК 616.71-001.5:539.4.014.1

\title{
ДВУХСЛОЙНАЯ МАТЕМАТИЧЕСКАЯ МОДЕЛЬ ШЕЙКИ БЕДРА ЧЕЛОВЕКА ДЛЯ ИССЛЕДОВАНИЯ НАПРЯЖЁННОГО СОСТОЯНИЯ ПРИ АРМИРОВАНИИ ИМПЛАНТАТАМИ РАЗЛИЧНОЙ КОНСТРУКЦИИ
}

\section{А. В. Нехожин}

Самарский государственный технический университет, Россия, 443100, Самара, ул. Молодогвардейская, 244.

E-mail: stswoon@yandex.ru

\begin{abstract}
Представлена двухслойная математическая модель шейки бедренной кости человека, армированная имплантатами различной конструкции. Спроектированы новые модели имплантатов. Разработано новое программное обеспечение для геометрического моделирования костной ткани с внедрёнными имплантатами. Предложена рациональная геометрия по внедрению имплантатов в костную тканъ для максимальной разгрузки наиболее нагруженных областей. Решён ряд краевых задач по оценке напряжённо-деформированного состояния в армированной шейке бедра. Показано, что в армированной конструкции напряженное состояние в наиболее нагруженной области значительно меньше, чем в неармированной.
\end{abstract}

Ключевые слова: бедренная кость, кортикальная и губчатая костная ткань, имплантат, армирование, геометрическая модель, метод конечных элементов, напряженно-дебормированное состояние.

Введение. В работе [1] предложена хирургическая методика профилактики переломов шейки бедренной кости путём принудительного введения имплантатов из различных материалов.

В работе [2] проведено моделирование напряжённо-деформированного состояния и получены положительные результаты, показывающие эффективность использования имплантатов для уменьшения вероятности перелома кости. Однако в [2] была рассмотрена лишь однослойная модель бедренной кости, которая фактически имеет более сложную структуру, в частности во многих работах рассматривается двухслойная модель $[3,4]$, состоящая из кортикальной кости - внешний слой и губчатой кости - внутренний слой.

В исследовании [2] использовалась геометрия кости проекта The Titanium Bone Project, полученная при помощи лазерного сканирования [5-7], которая упрощалась авторами в CAD-приложении KOMПAC (kompas.ru). Для экспорта упрощённой модели в конечно-элементный комплекс ANSYS (www.ansys. com) была специально разработана программа, которая по модели из CAD КОМПАС генерировала код APDL (ANSYS Parametric Design Language) для построения конечно-элементного разбиения, которое и использовалось для расчёта напряжённо-деформированного состояния кости.

1. Геометрическое моделирование. Поскольку для построения двухслойной модели шейки бедренной кости человека необходима её более точная геометрия, в данной статье предлагается другой подход к её построению, нежели

Анатолий Вадимович Нехожин, аспирант, каф. прикладной математики и информатики. 
в работе [2]. Ключевые особенности этого подхода:

- использование алгоритма автоматического упрощения геометрии кости;

- значительное снижение времени построения геометрии кости;

- возможность регулировать точность построения геометрии.

В качестве алгоритма автоматического упрощения геометрии кости проекта The Titanium Bone Project ${ }^{1}$, было решено использовать алгоритм Quadric Edge Collapse Decimation (QECD), который позволяет упростить модель без нарушения топологии. Для этой цели был выбран программный продукт MeshLab (meshlab.sourceforge.net), который представляет собой набор различных фильтров для работы с объёмными моделями. Один из фильтров для этой программы и реализует необходимый алгоритм. В результате применения фильтра получаем упрощенную геометрическую модель. Её можно сохранить в формате VRML, спецификация которого находится по адресу www . web3d.org/x3d/specifications/vrml/ISO-IEC-14772-VRML97. На языке Python (www.python.org) был разработан скрипт, который позволяет конвертировать данные из формата VRML в код APDL.

Таким образом, алгоритм построения модели кости можно описать так:

1) открытие объемной модели в программе MeshLab;

2) последовательное применение фильтра QECD до достижения необходимого соотношения точности и простоты модели;

3) конвертирование данных при помощи Python-скрипта.

На рис. 1 представлена геометрия кости после применения фильтра QECD к модели кости проекта The Titanium Bone Project.

Для построения двухслойной модели шейки бедренной кости из геометрической модели кости, полученной с использованием фильтра QECD, paзработана вспомогательная программа, которая преобразует исходную модель в двухслойную. Основная идея этой программы - создание внутреннего слоя кости за счёт уменьшения масштаба исходной геометрии с учётом невыпуклой особенности геометрии кости. Для построения двухслойной модели кости использовалась геометрическая модель, представленная на рис. 1, а.

Для автоматизации «внедрения» имплантатов в шейку бедренной кости человека и генерации конечно-элементной сетки разработаны вспомогательные программные средства со следующими особенностями:

- генерация APDL-команд для построения геометрии имплантатов по заданным характеристикам (длина, диаметр, шаг резьбы и прочее);

- позиционирование имплантата внутри шейки бедренной кости;

- генерация APDL-команд для объединения геометрий кости и имплантата;

- задание характеристик материала и типа конечных элементов для кости и имплантата.

На рис. 2 показано расположение имплантатов различного вида в шейке бедренной кости. Были использованы как «старые» имплантаты [2]: спица, штопор [8], так и ранее не участвующий в исследовании [2] шнековый винт [8].

2. Краевые условия и механические характеристики материалов. На рис. 3 схематически представлены внешние нагрузки и закрепления бедренной кости, соответствующие падению человека с высоты своего роста. Сосредоточенная нагрузка $F$ равномерно распределена по части поверхности головки

\footnotetext{
${ }^{1}$ VRML-модель кости проекта The Titanium Bone Project, полученная лазерным сканированием, находится по адресу webspace.utexas.edu/reyesr/titanium/3D_models.html.
} 


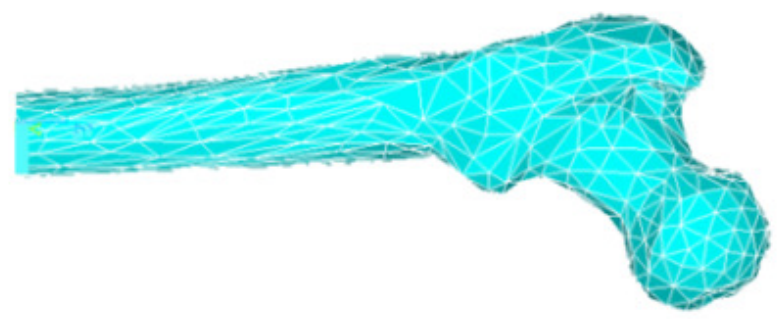

a

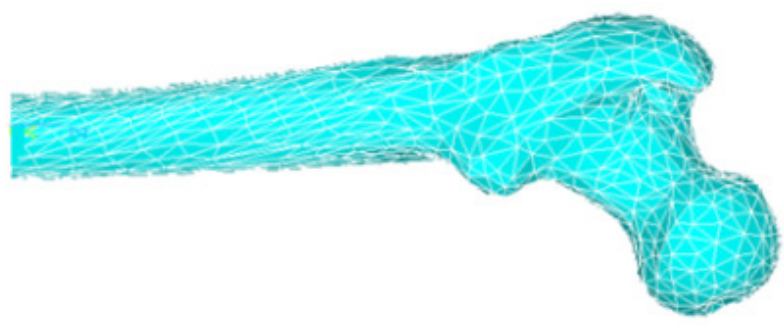

б

Рис. 1. Геометрия кости (показана верхняя часть) после применения фильтра QECD к модели кости проекта The Titanium Bone Project: a-938 точек; б-1875 точек

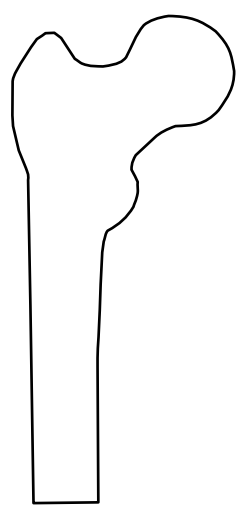

a

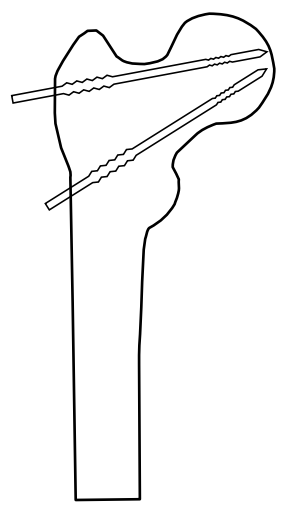

ДI

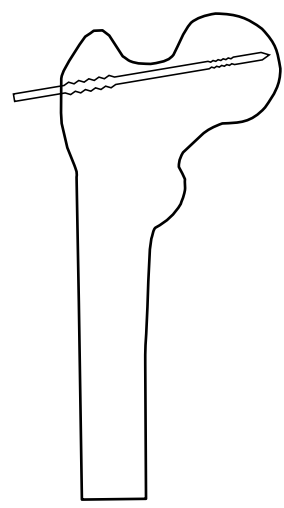

$\sigma$

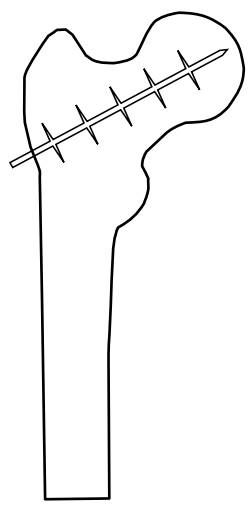

e

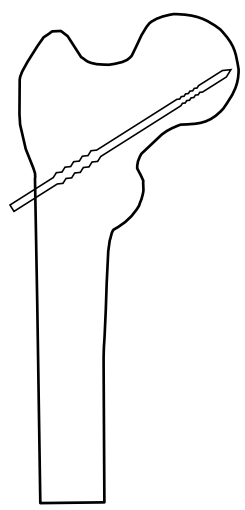

$B$

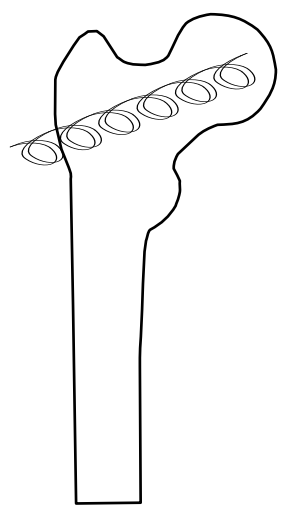

ЖK

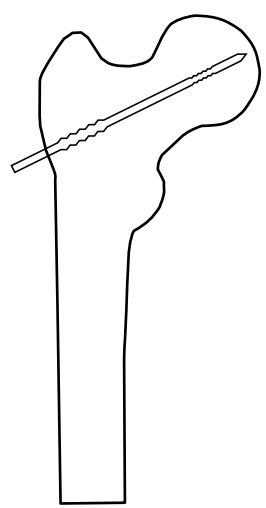

$\Gamma$

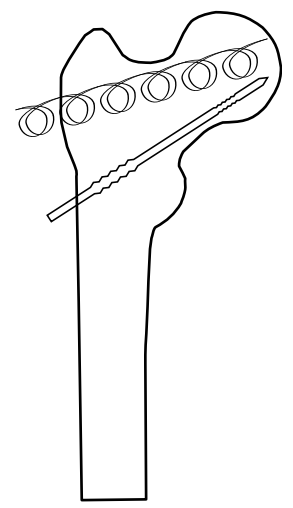

3

Рис. 2. Расположение имплантатов в шейке бедренной кости: $а$ - неармированная кость; бспица сверху; в-спица снизу; г- спица посередине; д - спица - спица; е-шнек; ж-штопор; 3 штопор - спица 


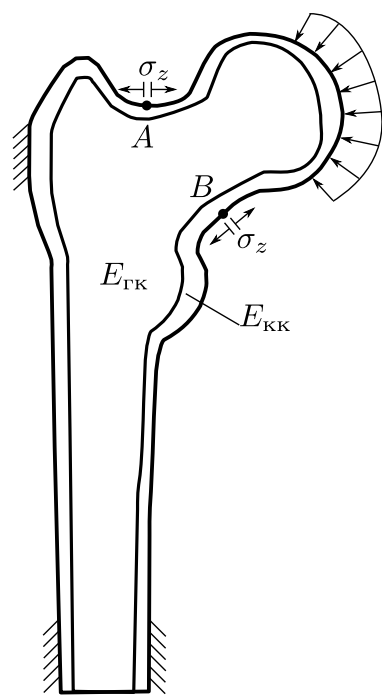

Рис. 3. Схема двухслойной модели бедренной кости; нагрузки и закрепления кости при падении человека бедренной кости таким образом, чтобы интегральная величина от распределённой нагрузки численно была равна значению $F$. Равнодействующая распределённых сил $F$ в расчётах принималась равной 8000 Н. Такое значение $F$ использовалось в реальных экспериментальных исследованиях [9].

Необходимо отметить, что в работе [2] использовалась однослойная модель кости с усреднёнными механическими характеристиками нелинейной (упругопластической) диаграммы деформирования материала кости, приведённой в [10]. Однако для двухслойной модели кости не удалось найти аналогичных нелинейных диаграмм механических характеристик для губчатого и кортикальных слоёв кости. Поэтому в данной статье использованы изотропные однородные характеристики кости в упругой области [4] и титановых имплантатов, численные значения которых представлены в табл. 1.

Таблица 1

Механические характеристики материалов

\begin{tabular}{l|c|c}
\hline \multicolumn{1}{c|}{ Материал } & Модуль Юнга, Па & Коэффициент Пуассона \\
\hline Кортикальная кость & $1,7 \cdot 10^{10}$ & 0,32 \\
Губчатая кость & $3,25 \cdot 10^{9}$ & 0,29 \\
Титан & $1,1 \cdot 10^{11}$ & 0,32 \\
\hline
\end{tabular}

3. Конечно-элементное разбиение и результаты расчётов. Разбиение модели на конечные элементы проводится аналогично работе [2] с тем лишь различием, что расстояние $D$ между узлами конечного элемента было выбрано в соответствии с данными работ $[11,12]$, где указано, что среднее расстояние между центрами остеонов (клетки кости, из которых состоит её матрица) в физической структуре костной ткани имеет значение $D=0,3$ мм.

В работе [2] доказаны следующие положения:

1) максимальные нормальные компоненты тензора напряжений (по модулю) на поверхности больше соответствующих напряжений внутри кости;

2) в конструкции шейки бедра имеются приповерхностные (критические) области, в которых нормальные компоненты имеют максимальные (по модулю) значения;

3) итерационный процесс решения краевой (нелинейной) задачи является сходящимся, что установлено выполненными расчётами со сгущением конечно-элементной сетки.

На основании этих результатов в данной работе проведено исследование только критических областей. Исследуются верхняя и нижняя части шейки бедра (точки $A$ и $B$ соответственно, см. рис. 3).

Следует отметить, что согласно [13] нормальные напряжения растяжения 
(точка $B$ на рис. 3) более опасны (до 2 раз), чем напряжения сжатия.

Для анализа напряжённого состояния в приповерхностных наиболее нагруженных областях (точки $A$ и $B$ на рис. 3) введены локальные системы координат, при этом ось $O z$ имеет направление касательной в выбранном сечении (см. рис. 3), и в этом же направлении возникают максимальные (по модулю) нормальные напряжения $\sigma_{z}$, которые в дальнейшем и были подвержены тщательному анализу при решении краевых задач с различной конструкцией имплантатов.

В табл. 2 приведены систематизированные результаты расчётов компоненты $\sigma_{z}$ (по модулю) в точках $A$ и $B$ для разных типов имплантатов, а в колонках $\Delta \sigma_{z}$ показано, на сколько (в процентах) уменьшилась (по модулю) величина $\sigma_{z}$ в конструкциях с имплантатами по сравнению с этой же конструкцией без имплантатов.

Таблица 2

Максимальные (по модулю) значения $\sigma_{z}$ в областях сжатия (точка $\boldsymbol{A}$ ) и растяжения (точка $B$ )

\begin{tabular}{l|c|c|c|c}
\hline \multirow{2}{*}{\multicolumn{1}{c|}{ Имплантат }} & \multicolumn{2}{|c|}{ точка $A$} & \multicolumn{2}{c}{ точка $B$} \\
\cline { 2 - 5 } & $\sigma_{z}$, Па & $\Delta \sigma_{z}, \%$ & $\sigma_{z}$, Па & $\Delta \sigma_{z}, \%$ \\
\hline Неармированная кость & $1,64 \cdot 10^{8}$ & - & $6,57 \cdot 10^{7}$ & - \\
Спица вверху & $1,49 \cdot 10^{8}$ & 10,1 & $6,39 \cdot 10^{7}$ & 2,8 \\
Спица внизу & $1,66 \cdot 10^{8}$ & $-1,2$ & $6,10 \cdot 10^{7}$ & 7,7 \\
Спица - спица & $1,47 \cdot 10^{8}$ & 11,6 & $5,86 \cdot 10^{7}$ & 12,1 \\
Спица посередине & $1,60 \cdot 10^{8}$ & 2,5 & $6,49 \cdot 10^{7}$ & 1,2 \\
Шнек & $1,64 \cdot 10^{8}$ & 0,0 & $6,47 \cdot 10^{7}$ & 1,5 \\
Штопор & $1,66 \cdot 10^{8}$ & $-1,2$ & $6,32 \cdot 10^{7}$ & 4,0 \\
Штопор - спица & $1,69 \cdot 10^{8}$ & $-3,2$ & $5,96 \cdot 10^{7}$ & 10,2 \\
\hline
\end{tabular}

Анализ данных табл. 2 свидетельствует о том, что для снижения уровня нагрузки наиболее рациональной конструкцией (для данного вида нагрузки) является система «кость - спица - спица». В технологическом смысле это также и оптимальный вариант превентивного «лечения» костной ткани.

Выводы. В данной статье предложена новая методика построения геометрии бедренной кости, разработаны необходимые программные инструменты и спецификации, реализована программа для конвертирования данных в необходимый формат. Показано превосходство новой методики над имеющимся подходом к построению геометрии бедренной кости. Более точно учтены геометрические и механические характеристики кости. Проанализированы результаты и выбрана оптимальная модель имплантирования - «кость спица - спица». По результатам расчётов такая конструкция позволяет снизить наибольшие напряжения в кости примерно на $12 \%$.

\section{БИБЛИОГРАФИЧЕСКИЙ СПИСОК}

1. А. Л. Матвеев, Оперативный способ профилактики переломов шейки бедренной кости: Патент РФ на на изобретение № 2316280 от 10.02.2008. [A. L. Matveev, Operative way of femoral neck fractures preventing: Invention patents of the Russian Federation. No. 2316280 of February 10, 2008].

2. В. П. Радченко, А. В. Нехожин, А. Л. Матвеев, "Математическое моделирование напряжённого состояния армированной костной ткани шейки бедра при статических на- 
грузках" // Вестн. Сам. гос. техн. ун-та. Сер. Физ.-мат. науки, 2011. № 4(25). С. 7581. [V. P. Radchenko, A. V. Nekhozhin, A. L. Matveev, "Mathematical modeling of the stress state of reinforced bone tissue of the femoral neck with static load" // Vestn. Samar. Gos. Tekhn. Univ. Ser. Fiz.-Mat. Nauki, 2011. no.4(25). Pp. 75-81].

3. Ю. И. Афанасъев, Н. А. Юрина, Е. Ф. Котовский, Гистология. М.: Медицина, 2002. 774 c. [Yu. I. Afanas'yev, N. A. Yurina, Ye. F. Kotovskiy, Histology. M.: Meditsina, 2002. 774 pp.]

4. Г. И. Рогожников, С. Г. Конюхова, Ю. И. Няиин, С. А. Чернопазов, С. В. Еремина, "Влияние модуля упругости губчатой и кортикальной кости на напряженное состояние в области пластинчатого имплантата при окклюзионной нагрузке" // Российский журнал биомеханики, 2004. T. 8, № 1. C. 54-60. [G. I. Rogozhnikov, S. G. Konyuhova, Yu. I. Nyashin, S. A. Chernopazov, S. V. Eremina, "The influence of elasticity modulus of spongy and cortical bone on stress state near planar implant under occlusal load" // Russian Journal of Biomechanics, 2004. Vol. 8, no. 1. Pp. 54-60].

5. N. Harlan, "Titanium Bone Implants" // Materials Technology, 2000. Vol. 15, no. 3. Pp. 185187.

6. N. Harlan, R. Reyes, D. L. Bourell, J. J. Beaman, "Titanium Castings using Laser Scanned Data and Selective Laser Sintered Zirconia Molds" // ASM Journal of Materials Engineering E Performance, 2001. Vol. 10, no. 4. Pp. 410-413.

7. N. Harlan, R. Reyes, D. L. Bourell, "Building Better Bones"// Foundry Management \& Technology, 2000. Vol. 128, no. 8. Pp. 82-83.

8. А. Л. Матвеев, А. В. Нехожин, И. И. Матвеева, "Имплантаты для армирования шейки бедренной кости с целью профилактики переломов при остеопорозе" / В сб.: Aкmyальные вопросы диагностики, лечения и реабилитации болъных. Пенза, 2011. С. 160162. [A. L. Matveyev, A. V. Nekhozhin, I. I. Matveyeva, "Implants for reinforcement of the femoral neck to prevent fractures in osteoporotic" / In: Actual problems of diagnosis, treatment and rehabilitation of patients. Penza, 2011. Pp. 160-162].

9. Б. ШІ. Минасов, М. Ю. Ханин, Р. Р. Якупов, Т. Б. Минасов, "Результаты стендовых испытаний системы кость-имплантат-кость в условиях стандартного остеосинтеза при переломах проксимального отдела бедра"// Казанский медицинский журнал, 2010. № 1. C. 40-44. [B. Sh. Minasov, M. Yu. Khanin, P. P. Yakupov, T. B. Minasov, "The results of bone - implant - bone bench tests of standard osteosynthesis during fractures of the proximal part of the thigh bone" // Kazanskiy Medicinskiy Zhurnal, 2010. Vol. 91, no. 1. Pp. 40-44].

10. Вини, $X$. , "Изменение механических свойств компактной костной ткани человека в зависимости от возраста" // Механика полимеров, 1975. № 11. С. 659-663; англ. пер.: H. Vinz, "Change in the mechanical properties of human compact bone tissue upon aging" // Mechanics of Composite Materials, 1975. Vol.11, no.4. Pp. 568-571.

11. A. А. Утенъкин, "Кость - многоэтажный композит" // Химия и жизнъ, 1981. № 4. C. 38-40. [A. A. Uten'kin, "Bone is a multi-storey composite" // Khimiya i Zhizn', 1981. no. 4. Pp. 38-40].

12. Т. Б. Минасов, А. Е. Стрижков, Л. М. Бакусов, Р. В. Насыров, Структурная самоорганизация костной ткани и ее механизмы как источник диагностической информации. Уфa, 2010. 116 c. [T. B. Minasov, A. E. Strizhkov, L. M. Bakusov, R. V. Nasyrov, Structural self-organization of bone tissue and its mechanisms as a source of diagnostic information. Ufa, 2010. $116 \mathrm{pp}$.]

13. Р. Лайуни, "К вопросу о механических свойствах костной ткани" / В сб.: Физическое воспитание студентов творческих специальностей, Т. 4. Харьков, 2002. С. 1822. [R. Liayouni, "To a question on mechanical charecteristics bone tissue" / In: Physical education of students of creative disciplines, 4. Khar'kov, 2002. Pp. 18-22].

Поступила в редакцию 01/VII/2013;

в окончательном варианте - 22/VIII/2013. 
MSC: 97M60; 74L15, 74S05

\section{BILAYER MATHEMATICAL MODEL OF HUMAN FEMUR NECK FOR RESEARCH OF THE STRESS STATE AFTER REINFORCEMENT WITH DIFFERENT DESIGNS OF IMPLANTS}

\section{A. V. Nekhozhin}

Samara State Technical University,

244, Molodogvardeyskaya st., Samara, 443100, Russia.

E-mail: stswoon@yandex.ru

A bilayer mathematical model of human femur neck reinforced with implants of various designs is represented. New models of implants are designed. The software for geometric modeling of bone with the implant is developed. The rational geometry for placing implants into the bone tissue to unload the most loaded areas is proposed. A number of boundary value problems for evaluating the stress-strain state in the reinforced femoral neck are solved. It is shown that stress state in the most loaded region in the reinforced construction is considerably less than in the non-reinforced one.

Key words: femur, cortical and cancellous bone tissue, implant, reinforcement, geometric modeling, finite element method, stress-strain state, mechanical properties of bone.

Original article submitted 01/VII/2013; revision submitted 22/VIII/2013.

Anatoliy V. Nekhozhin, Postgraduate Student, Dept. of Applied Mathematics \& Computer Science. 